\title{
Annual Recurrences of Viral Hemorrhagic Septicemia Epizootics in Age 0 Pacific Herring Clupea pallasii Valenciennes, 1847
}

\author{
Paul K. Hershberger ${ }^{1, *}$, Theodore R. Meyers ${ }^{2}{ }^{\circledR}$, Jacob L. Gregg ${ }^{1}$, Maya L. Groner ${ }^{1,3}$, Sophie A. Hall ${ }^{1}$, \\ Hiruni T. Jayasekera ${ }^{1}$, Ashley H. MacKenzie ${ }^{1}$, Abigail S. Neat ${ }^{1}$, Ella N. Piatt ${ }^{1}$ and Kyle A. Garver ${ }^{4}$
}

1 Marrowstone Marine Field Station, Western Fisheries Research Center, U.S. Geological Survey, Nordland, WA 98358, USA; jgregg@usgs.gov (J.L.G.); mgroner@usgs.gov (M.L.G.); sahall@usgs.gov (S.A.H.); hirunijayasekera97@gmail.com (H.T.J.); amackenzie@usgs.gov (A.H.M.); abbeysneat@gmail.com (A.S.N.); ellanpiatt@gmail.com (E.N.P.)

2 Juneau Fish Pathology Laboratory, Alaska Department of Fish \& Game, P.O. Box 115526, Juneau, AK 99811, USA; ted.meyers@alaska.gov

3 Prince William Sound Science Center, Cordova, AK 99574, USA

4 Pacific Biological Station, Fisheries and Oceans Canada, Nanaimo, BC V9T 6N7, Canada; kyle.garver@dfo-mpo.gc.ca

* Correspondence: phershberger@usgs.gov

Citation: Hershberger, P.K.; Meyers, T.R.; Gregg, J.L.; Groner, M.L.; Hall, S.A.; Jayasekera, H.T.; MacKenzie, A.H.; Neat, A.S.; Piatt, E.N.; Garver, K.A. Annual Recurrences of Viral Hemorrhagic Septicemia Epizootics in Age 0 Pacific Herring Clupea pallasii Valenciennes, 1847. Animals 2021, 11, 2426. https://doi.org/10.3390/ ani11082426

Academic Editors: Mohamed Faisal and Jun Li

Received: 12 July 2021

Accepted: 9 August 2021

Published: 18 August 2021

Publisher's Note: MDPI stays neutral with regard to jurisdictional claims in published maps and institutional affiliations.

Copyright: (C) 2021 by the authors Licensee MDPI, Basel, Switzerland. This article is an open access article distributed under the terms and conditions of the Creative Commons Attribution (CC BY) license (https:// creativecommons.org/licenses/by/ $4.0 /)$.
Simple Summary: Pathogen surveillances in wild marine fish populations need to be carefully designed to address specific research or management objectives. Surveillance strategies should be designed around host life history characteristics, host and pathogen geographic ranges, laboratory diagnostic tools that are specific to the epidemiology of each pathogen, and the goal of the surveillance program. We demonstrate how the potential impacts of viral hemorrhagic septicemia can be underappreciated in populations of Pacific herring by comparing results from opportunistically collected samples with those from more targeted epidemiological investigations that were focused on times and locations with high disease probability.

Abstract: Throughout a 20 year biosurveillance period, viral hemorrhagic septicemia virus was isolated in low titers from only 6/7355 opportunistically sampled adult Pacific herring, reflecting the typical endemic phase of the disease when the virus persists covertly. However, more focused surveillance efforts identified the presence of disease hot spots occurring among juvenile life history stages from certain nearshore habitats. These outbreaks sometimes recurred annually in the same temporal and spatial patterns and were characterized by infection prevalence as high as $96 \%$. Longitudinal sampling indicated that some epizootics were relatively transient, represented by positive samples on a single sampling date, and others were more protracted, with positive samples occurring throughout the first 10 weeks of the juvenile life history phase. These results indicate that viral hemorrhagic septicemia (VHS) epizootics in free-ranging Pacific herring C. pallasii are more common than previously appreciated; however, they are easily overlooked if biosurveillance efforts are not designed around times and locations with high disease potential.

Keywords: VHS; viral hemorrhagic septicemia; Pacific herring; epizootic

\section{Introduction}

Viral hemorrhagic septicemia virus, genogroup IVa (hereafter referred to as VHS virus) has a broad host range in marine fishes throughout the coastal waters of North America and Asia, including China, Japan, and Korea [1-3]. Throughout parts of this range, the ecology of the virus is closely tied to Pacific herring (Clupea pallasii Valenciennes, 1847) and other hosts [4] which are highly susceptible to the resulting disease (viral hemorrhagic septicemia; VHS) and serve as effective reservoirs between outbreaks [5]. All available 
data indicate that apparently healthy hosts are responsible for virus perpetuation during these typical endemic periods via persistence in fully convalesced individuals [5]. Further, the reservoir of VHS virus in marine forage fishes such as Pacific herring C. pallasii and Pacific sardine represents a source of viral spillover to cultured Atlantic salmon in marine pet pens in Washington (USA) and British Columbia (Canada), which experience periodic VHS epizootics [3].

Epizootics of VHS periodically occur in wild marine fishes [1-3], presumably as a result of alignment between permissive environmental conditions and sympatry of host reservoirs and susceptible species. It is important to recognize that most epizootics go unnoticed because they remain small in scale and/or they occur in remote locations that are difficult to sample. Because of these logistical challenges, reports of VHS epizootics in wild marine fishes generally describe single sample collections from reported fish kills. As such, neither the temporal and spatial patterns nor the complete progression of natural VHS epizootics has been properly documented in wild marine fishes. Interestingly, although VHS virus occurs in Pacific herring C. pallasii from Puget Sound, Washington, USA, where the disease sometimes occurs in confined wild cohorts [6,7], epizootics among free-ranging herring have not yet been reported from this region [8].

This study was performed to document the interannual occurrence of VHS epizootics in Pacific herring C. pallasii from the same geographic locations in consecutive years and to document the progression of a single epizootic throughout the disease onset, peak, and recovery phases. To emphasize the ease with which these epizootics can be overlooked, we also report the results from 20 years of VHS virus surveillances in Pacific herring C. pallasii populations throughout the NE Pacific Ocean, including annual sampling from pre-spawn aggregations in Prince William Sound and Sitka Sound as well as opportunistic sampling from other locations.

\section{Materials and Methods}

Pacific herring C. pallasii ( $n=7355$ ) were collected from locations throughout coastal areas of the NE Pacific Ocean using various gear types and sampled for presence of VHS virus (Table 1). Spring samples (March-April) from Prince William Sound and Sitka Sound, AK consisted of pre-spawn or actively spawning fish that were collected in conjunction with state stock assessment surveys. Other collections were largely opportunistic, but some were expanded to targeted surveillances when possible. In accordance with requirements of the World Organization for Animal Health (OIE) for epidemiological surveillance, efforts were made to analyze 60 fish from each sample collection, as this sample size is appropriate for detecting an agent with $5 \%$ minimum expected prevalence. The VHS virus infection status was determined by plaque assay [9] of fresh or previously frozen $\left(-80{ }^{\circ} \mathrm{C}\right.$ unless noted) kidney/spleen pools on polyethylene glycol-pretreated [10] monolayers of epithelioma papulosum cyprini cells $[11,12]$ in 24-well plates; the minimum detection threshold was 400 plaque forming units/g. Any samples demonstrating questionable cytopathic effects below this threshold were subjected to additional blind passage onto fresh EPC cells. VHS virus was confirmed in cell culture-positive samples using a TaqMan-based reverse transcription PCR (RT-qPCR) targeting the N-gene and sequencing to determine virus type and subtype [13].

More targeted investigations were launched in nearshore areas including embayments and boat harbors that were presumed hot spots for VHS (Table 2). Additionally, longitudinal sampling of juvenile Pacific herring C. pallasii from Port Angeles Harbor, WA, USA, occurred approximately every 2 weeks from 18 September to 5 November 2018, from 23 July to 24 September 2019, and from 23 June to 20 October 2020 (Figure 1A); analogous longitudinal sampling occurred from Port Ludlow Harbor, WA, USA, 25 July-25 September 2020. Samples were collected by cast net and consisted of 22-60 fish/day. All kidney/spleen samples were frozen at $-70{ }^{\circ} \mathrm{C}$ (with the exception of 2014 samples from Puget Sound, which were frozen at $-20^{\circ} \mathrm{C}$ ), then processed by viral plaque assay and viral isolates were confirmed as before. 
Table 1. Voral hemorrhagic septicemia (VHSV) virus survey results from Pacific herring C. pallasii during typical endemic periods.

\begin{tabular}{|c|c|c|c|c|c|c|c|}
\hline Year & Herring Stock & Collection Site & Collection Date & Gear Type & $\begin{array}{c}\text { Adult/ } \\
\text { Juvenile (A/J) }\end{array}$ & $\begin{array}{c}\text { Mean Fork Length } \\
\text { mm (SD) }\end{array}$ & $\begin{array}{c}\text { VHSV Detection by } \\
\text { Cell Culture } \\
\text { (No. Positive/ } n \text { ) }\end{array}$ \\
\hline 2001 & Cook Inlet, AK, USA & Kachemak Bay & 18 June & Purse Seine & $\mathrm{J}$ & NA & $0 \%(0 / 32)$ \\
\hline \multirow[t]{4}{*}{2003} & Puget Sound, WA, USA & Port Orchard & 6 February & Trawl & A & $179(19)$ & $0 \%(0 / 60)$ \\
\hline & & Port Madison & 6 February & Trawl & A & $173(19)$ & $0 \%(0 / 60)$ \\
\hline & & Skagit Bay & 12 February & Trawl & A & $161(10)$ & $0 \%(0 / 60)$ \\
\hline & & Port Susan & 12 February & Trawl & A & $171(14)$ & $0 \%(0 / 60)$ \\
\hline \multirow[t]{3}{*}{2004} & Puget Sound, WA, USA & Cherry Point & 4 May & Trawl & A & $221(16)$ & $1.7 \%(1 / 60)$ \\
\hline & N. Hecate Strait, AK, USA & Behm Canal & 11 April & Purse Seine & $\mathrm{A}$ & NA & $0 \%(0 / 20)$ \\
\hline & Frederick Sound, AK, USA & Hidden Falls & 9 April & Cast Net & $\mathrm{J}$ & NA & $0 \%(0 / 15)$ \\
\hline 2005 & Cook Inlet, AK, USA & Dry Bay & 16 May & Purse Seine & $\mathrm{A}$ & NA & $0 \%(0 / 53)$ \\
\hline \multirow[t]{3}{*}{2006} & Cook Inlet, AK, USA & Nordyke Bay & 18 May & Purse Seine & A & NA & $0 \%(0 / 60)$ \\
\hline & & Oil Bay & 21 May & Purse Seine & A & NA & $0 \%(0 / 60)$ \\
\hline & & Iniskin Bay & 22 May & Purse Seine & $\mathrm{A}$ & NA & $0 \%(0 / 60)$ \\
\hline \multirow[t]{9}{*}{2007} & & St. Matthews Bay & 5 April & Cast Net & $\mathrm{A}$ & $224(17)$ & $0 \%(0 / 60)$ \\
\hline & Prince William Sound, AK, USA & Simpson Bay & 19 April & Purse Seine & $\mathrm{J}$ & $86(6.3)$ & $0 \%(0 / 60)$ \\
\hline & & Simpson Bay & 2 December & Purse Seine & $\mathrm{A}$ & $187(13)$ & $0 \%(0 / 60)$ \\
\hline & Sitka Sound, AK, USA & Cannon Island & 9 April & Cast Net & $\mathrm{A}$ & $215(18)$ & $0 \%(0 / 60)$ \\
\hline & Kodiak, AK, USA & Uganik Bay & 18 February & Purse Seine & A & NA & $0 \%(0 / 30)$ \\
\hline & & Uyak Bay & 21 February & Purse Seine & $\mathrm{A}$ & NA & $0 \%(0 / 35)$ \\
\hline & Cook Inlet, AK, USA & Iniskin Bay & 16 May & Purse Seine & $\mathrm{A}$ & NA & $1.7 \%(1 / 60)$ \\
\hline & & Amakdedulia Cove & 27 May & Purse Seine & $\mathrm{A}$ & NA & $1.7 \%(1 / 60)$ \\
\hline & & Ursus Cove & 27 May & Purse Seine & A & NA & $0 \%(0 / 60)$ \\
\hline \multirow[t]{7}{*}{2008} & Prince William & Unknown & 17 March & Purse Seine & $\mathrm{J}$ & $141(11)$ & $0 \%(0 / 60)$ \\
\hline & Sound, AK, USA & Fish Bay & 19 March & Purse Seine & $\mathrm{A}$ & $236(27)$ & $0 \%(0 / 45)$ \\
\hline & & Evans Point & 24 March & Purse Seine & $\mathrm{A}$ & $208(18)$ & $0 \%(0 / 60)$ \\
\hline & & Whale Bay & 24 March & Purse Seine & $\mathrm{J}$ & $149(22)$ & $0 \%(0 / 60)$ \\
\hline & & St. Matthews Bay & 8 April & Purse Seine & $\mathrm{A}$ & NA & $0 \%(0 / 32)$ \\
\hline & & Port Gravina & 8-12 November & Purse Seine/Jig & $\mathrm{A}$ & $197(23)$ & $0 \%(0 / 80)$ \\
\hline & Sitka Sound, AK, USA & N. Middle Island & 26 March & Purse Seine & $\mathrm{A}$ & $249(14)$ & $0 \%(0 / 60)$ \\
\hline \multirow[t]{2}{*}{2009} & & Port Gravina & 20 March & Purse Seine & $\mathrm{A}$ & $199(15)$ & $0 \%(0 / 60)$ \\
\hline & Prince William Sound, AK, USA & Port Gravina & 20 March & Purse Seine & $\mathrm{A}$ & $168(11)$ & $0 \%(0 / 60)$ \\
\hline
\end{tabular}


Table 1. Cont.

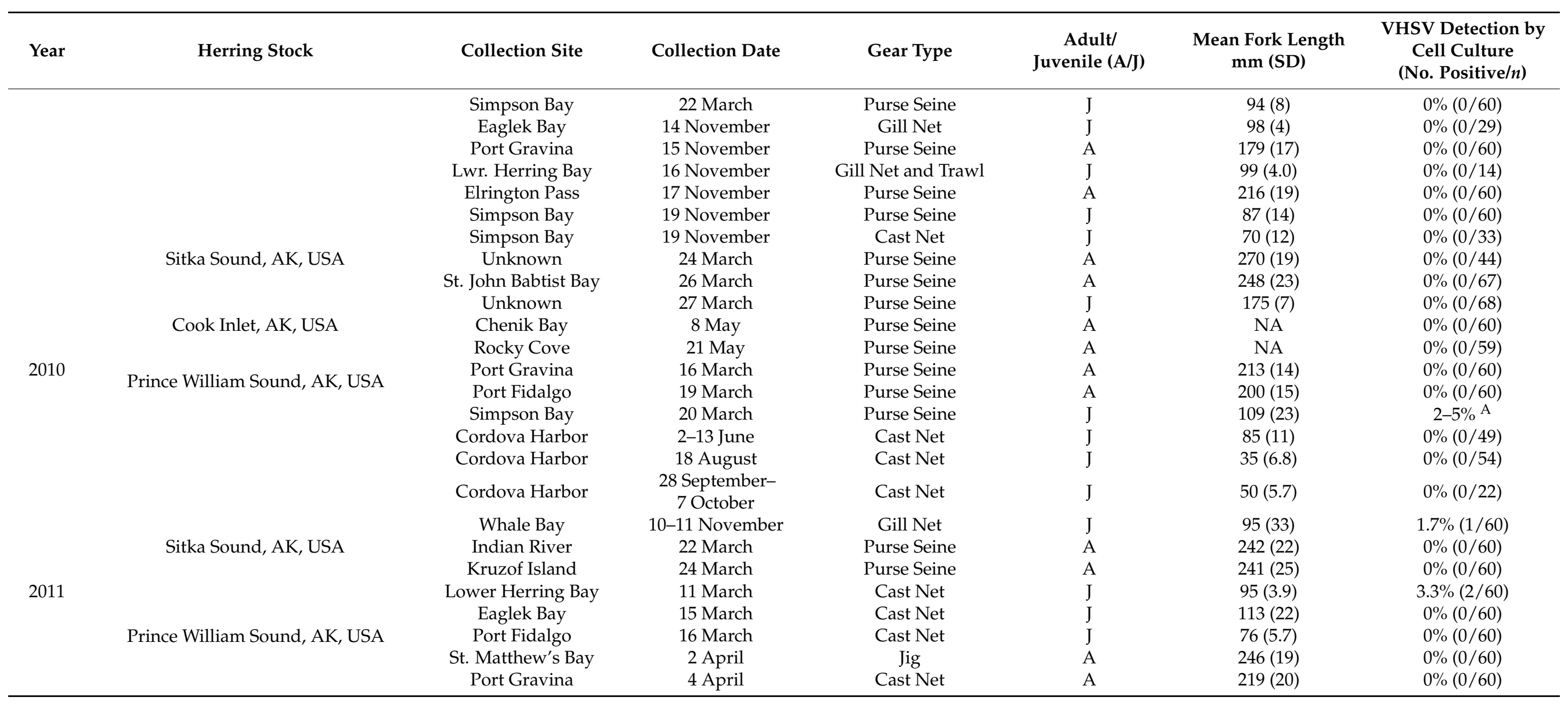


Table 1. Cont.

\begin{tabular}{|c|c|c|c|c|c|c|c|}
\hline Year & Herring Stock & Collection Site & Collection Date & Gear Type & $\begin{array}{c}\text { Adult/ } \\
\text { Juvenile (A/J) }\end{array}$ & $\begin{array}{c}\text { Mean Fork Length } \\
\text { mm (SD) }\end{array}$ & $\begin{array}{c}\text { VHSV Detection by } \\
\text { Cell Culture } \\
\text { (No. Positive/ } n \text { ) }\end{array}$ \\
\hline \multirow{23}{*}{2012} & \multirow{8}{*}{$\begin{array}{c}\text { Sitka Sound, AK, USA } \\
\text { Cook Inlet, AK, USA }\end{array}$} & Port Fidalgo & 6 April & Purse Seine & A & $253(12)$ & $0 \%(0 / 60)$ \\
\hline & & Simpson Bay & 15 November & Cast Net & $\mathrm{J}$ & $60(6.1)$ & $0 \%(0 / 60)$ \\
\hline & & Whale Bay & 20 November & Cast Net & $\mathrm{J}$ & $83(6.4)$ & $0 \%(0 / 60)$ \\
\hline & & Port Gravina & 21 November & Purse Seine & $\mathrm{A}$ & $205(19)$ & $0 \%(0 / 30)$ \\
\hline & & Port Gravina & 22 November & Purse Seine & $\mathrm{A}$ & $157(11)$ & $0 \%(0 / 30)$ \\
\hline & & Long Island & 22 March & Purse Seine & $\mathrm{A}$ & $232(16)$ & $0 \%(0 / 60)$ \\
\hline & & Bruin Bay & 4 May & Purse Seine & $\mathrm{A}$ & NA & $0 \%(0 / 60)$ \\
\hline & & Rocky Cove & 13 May & Purse Seine & $\mathrm{A}$ & $224(60)^{B}$ & $0 \%(0 / 60)$ \\
\hline & Bristol Bay, AK, USA & Togiak Bay & 9 May & Purse Seine & $\mathrm{A}$ & NA & $0 \%(0 / 60)$ \\
\hline & \multirow{11}{*}{ Prince William Sound, AK, USA } & Simpson Bay & 11 January & Cast Net & $\mathrm{J}$ & $57(2.8)$ & $0 \%(0 / 60)$ \\
\hline & & Glacier Island Pass & 8 February & Dip Net & $\mathrm{A}$ & $240(13)$ & $0 \%(0 / 15)$ \\
\hline & & Eaglek Bay & 21 March & Gill Net & $\mathrm{J}$ & $99(3.2)$ & $0 \%(0 / 30)$ \\
\hline & & Port Gravina & 28 March & Purse Seine & $\mathrm{A}$ & $218(16)$ & $0 \%(0 / 60)$ \\
\hline & & Port Gravina & 31 March & Purse Seine & $\mathrm{A}$ & $216(16)$ & $0 \%(0 / 60)$ \\
\hline & & Simpson Bay & 20 April & Cast Net & $\mathrm{J}$ & $78(16)$ & $0 \%(0 / 30)$ \\
\hline & & Port Gravina & 9 November & Cast Net & $\mathrm{J}$ & $63(5.5)$ & $0 \%(0 / 30)$ \\
\hline & & Simpson Bay & 9 November & Cast Net & $\mathrm{J}$ & $72(9.0)$ & $0 \%(0 / 30)$ \\
\hline & & Zaikoff Bay & 13 November & Cast Net & $\mathrm{J}$ & $72(4.4)$ & $0 \%(0 / 60)$ \\
\hline & & Lower Herring Bay & 15 November & Cast Net & $\mathrm{J}$ & $90(4.3)$ & $0 \%(0 / 30)$ \\
\hline & & Port Gravina & 15 November & Purse Seine & $\mathrm{A}$ & $159(14)$ & $0 \%(0 / 60)$ \\
\hline & \multirow[t]{3}{*}{ Sitka Sound, AK, USA } & N. Kasiana Isl. & 3 April & Cast Net & $\mathrm{A}$ & $233(22)$ & $0 \%(0 / 60)$ \\
\hline & & St. John Bay & 4 April & Purse Seine & A & $214(24)$ & $0 \%(0 / 60)$ \\
\hline & & Sitka Harbor & 4 April & Cast Net & $\mathrm{A}$ & $225(22)$ & $0 \%(0 / 60)$ \\
\hline \multirow{3}{*}{2013} & Cook Inlet, AK, USA & Bruin Bay & 7 May & Purse Seine & $\mathrm{A}$ & $254(10)^{B}$ & $0 \%(0 / 60)$ \\
\hline & \multirow{2}{*}{ Prince William Sound, AK, USA } & Port Gravina & 27 March & Purse Seine & $\mathrm{J}$ & $147(16)$ & $0 \%(0 / 60)$ \\
\hline & & Port Gravina & 31 March & Purse Seine & $\mathrm{A}$ & $232(20)$ & $0 \%(0 / 60)$ \\
\hline
\end{tabular}


Table 1. Cont.

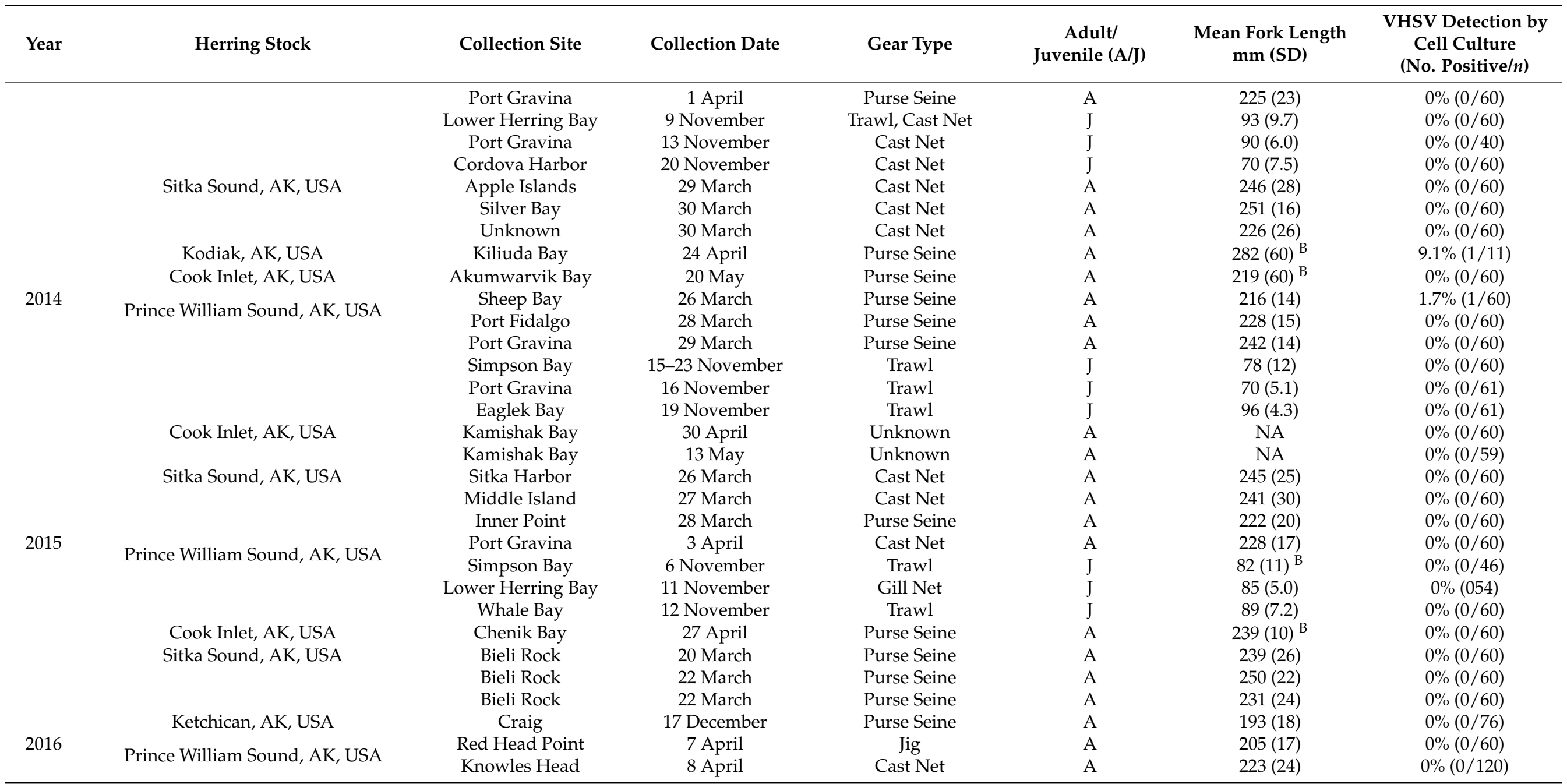


Table 1. Cont.

\begin{tabular}{|c|c|c|c|c|c|c|c|}
\hline Year & Herring Stock & Collection Site & Collection Date & Gear Type & $\begin{array}{c}\text { Adult/ } \\
\text { Juvenile (A/J) }\end{array}$ & $\begin{array}{c}\text { Mean Fork Length } \\
\text { mm (SD) }\end{array}$ & $\begin{array}{c}\text { VHSV Detection by } \\
\text { Cell Culture } \\
\text { (No. Positive/n) }\end{array}$ \\
\hline \multirow{10}{*}{2017} & \multirow{5}{*}{ Sitka Sound, AK, USA } & Simpson Bay & 29 October & Trawl & $\mathrm{J}$ & $82(3.5)$ & $0 \%(0 / 60)$ \\
\hline & & Lower Herring Bay & 2 November & Trawl & $\mathrm{J}$ & $96(4.4)$ & $0 \%(0 / 60)$ \\
\hline & & S. Salsbury Sound & 21 March & Purse Seine & $\mathrm{A}$ & $218(22)$ & $0 \%(0 / 60)$ \\
\hline & & North Crest & 22 March & Purse Seine & $\mathrm{A}$ & $215(13)$ & $0 \%(0 / 60)$ \\
\hline & & Point Brown & 22 March & Cast Net & $\mathrm{A}$ & $217(24)$ & $0 \%(0 / 60)$ \\
\hline & \multirow{2}{*}{$\begin{array}{l}\text { Prince William } \\
\text { Sound, AK, USA }\end{array}$} & Rocky Bay & 10 April & Purse Seine & $\mathrm{J}$ & $140(46)$ & $0 \%(0 / 60)$ \\
\hline & & Port Fidalgo & 10 April & Purse Seine & $\mathrm{A}$ & $191(14)$ & $0 \%(0 / 60)$ \\
\hline & \multirow[t]{3}{*}{ Sitka Sound, AK, USA } & Unknown & 24 March & Cast Net & $\mathrm{A}$ & $221(14)$ & $0 \%(0 / 60)$ \\
\hline & & S. Magoun Island & 25 March & Cast Net & $\mathrm{A}$ & $225(15)$ & $0 \%(0 / 60)$ \\
\hline & & Unknown & 25 March & Cast Net & $\mathrm{A}$ & $225(16)$ & $0 \%(0 / 60)$ \\
\hline \multirow[t]{4}{*}{2018} & \multirow{3}{*}{ Prince William Sound, AK, USA } & Port Fidalgo & 10-11 April & Purse Seine & $\mathrm{J}$ & $152(36)$ & $0 \%(0 / 60)$ \\
\hline & & Cedar Bay & 12 April & Purse Seine & $\mathrm{A}$ & $201(15)$ & $0 \%(0 / 60)$ \\
\hline & & Rocky Bay & 13 April & Purse Seine & $\mathrm{A}$ & $204(16)$ & $0 \%(0 / 60)$ \\
\hline & Sitka Sound, AK, USA & Guide Island & 22 March & Cast Net & $\mathrm{A}$ & $221(15)$ & $0 \%(0 / 56)$ \\
\hline \multirow[t]{7}{*}{2019} & \multirow[t]{2}{*}{ Puget Sound, WA, USA } & Oak Bay & 29 August & Dip Net & $\mathrm{J}$ & $103(3.7)$ & $0 \%(0 / 60)$ \\
\hline & & Double Bay & 5 April & Purse Seine & $\mathrm{A}$ & $176(14)$ & $0 \%(0 / 60)$ \\
\hline & \multirow[t]{2}{*}{ Prince William Sound, AK, USA } & Canoe Pass & 6 April & Purse Seine & $\mathrm{A}$ & $157(29)$ & $0 \%(0 / 60)$ \\
\hline & & Windy Bay & 6 April & Purse Seine & $\mathrm{A}$ & $179(13)$ & $0 \%(0 / 59)$ \\
\hline & \multirow[t]{3}{*}{ Sitka Sound, AK, USA } & Krestof Isl. & 25 March & Cast Net & $\mathrm{A}$ & $205(18)$ & $0 \%(0 / 60)$ \\
\hline & & Krestof Isl. & 26 March & Cast Net & $\mathrm{A}$ & $197(17)$ & $0 \%(0 / 60)$ \\
\hline & & Whitestone Narrows & 27 March & Cast Net & $\mathrm{A}$ & $204(18)$ & $0 \%(0 / 60)$ \\
\hline \multirow[t]{7}{*}{2020} & Puget Sound & Eagle Harbor & 26 August & Cast Net & $\mathrm{J}$ & $69(3.4)$ & $0 \%(0 / 60)$ \\
\hline & \multirow{2}{*}{ Prince William Sound, AK, USA } & Canoe Pass & 8 April & Purse Seine & $\mathrm{A}$ & $213(13)$ & $0 \%(0 / 130)$ \\
\hline & & Double Bay & 10 April & Purse Seine & $\mathrm{A}$ & $220(26)$ & $0 \%(0 / 59)$ \\
\hline & \multirow[t]{3}{*}{ Sitka Sound, AK, USA } & Kruzof & 31 March & Cast Net & $\mathrm{A}$ & $215(14)$ & $0 \%(0 / 60)$ \\
\hline & & Low Island & 1 April & Cast Net & $\mathrm{A}$ & $215(14)$ & $0 \%(0 / 60)$ \\
\hline & & Silver Bay & 2 April & Cast Net & $\mathrm{A}$ & $204(10)$ & $0 \%(0 / 60)$ \\
\hline & Kodiak, AK, USA & Uganik Bay & 21 April & Purse Seine & $\mathrm{A}$ & $199(10)^{B}$ & $0 \%(0 / 60)$ \\
\hline
\end{tabular}

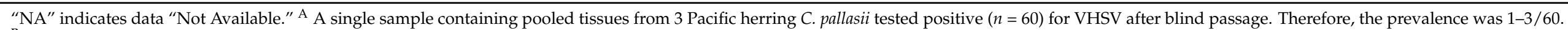

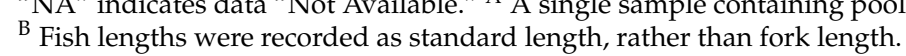


Table 2. Viral hemorrhagic septicemia virus (VHSV) survey results from Pacific herring C. pallasii during epizootic periods.

\begin{tabular}{|c|c|c|c|c|c|c|c|c|}
\hline Region & Year & Stock & Collection Site & Collection Date & Gear Type & $\begin{array}{l}\text { Adult/Juvenile } \\
\text { (A/J) }\end{array}$ & $\begin{array}{c}\text { Mean Fork } \\
\text { Length mm (SD) }\end{array}$ & $\begin{array}{l}\text { VHSV Detection by Cell Culture } \\
\text { (No. Positive/n) }\end{array}$ \\
\hline AK, USA & 2011 & Sitka Sound & Bear Cove Bay & 24 March & Cast Net & $\mathrm{J}$ & $108(11)$ & $63 \%(38 / 60)$ \\
\hline BC, Canada & 2018 & W. Vancouver Isl. & Hot Springs Cv. & 24 June & Dip Net & $\mathrm{J}$ & NA ${ }^{B}$ & $85 \%(22 / 26)$ \\
\hline \multirow[t]{5}{*}{ WA, USA } & 2014 & Puget Sound & Lopez Isl. A & 11 September & Beach Seine & $\mathrm{J}$ & $\mathrm{NA}^{\mathrm{C}}$ & $27 \%(6 / 22)$ \\
\hline & & Puget Sound & Waldron Isl. A & 12 September & Beach Seine & $\mathrm{J}$ & $\mathrm{NA}^{\mathrm{C}}$ & $13 \%(3 / 24)$ \\
\hline & 2018 & Puget Sound & Pt. Angeles Hbr. & 18 September- 5 November & Cast Net & $\mathrm{J}$ & $67(8.0)-78(7.4)$ & $6.7 \%(2 / 30)^{\mathrm{D}}$ \\
\hline & 2019 & Puget Sound & Pt. Ludlow Hbr. & 25 July-25 September & Cast Net & $\mathrm{J}$ & $52(2.4)-78(6.8)$ & $33 \%(20 / 60)^{D}$ \\
\hline & 2020 & Puget Sound & Pt. Angeles Hbr. & 23 June-20 October & Cast Net & $\mathrm{J}$ & $37(2.0)-63(8.3)$ & $63 \%(38 / 60)^{\mathrm{D}}$ \\
\hline
\end{tabular}

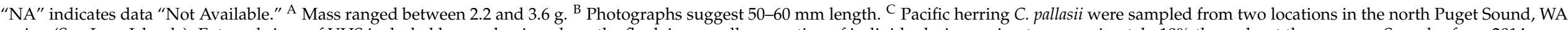

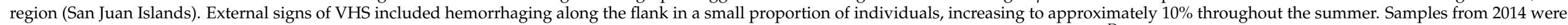

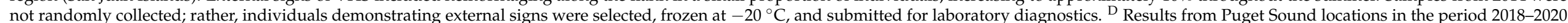
reflect the peak infection prevalence from annual longitudinal sampling at each site. Results from all sampling dates are depicted in Figure 1A,B. 

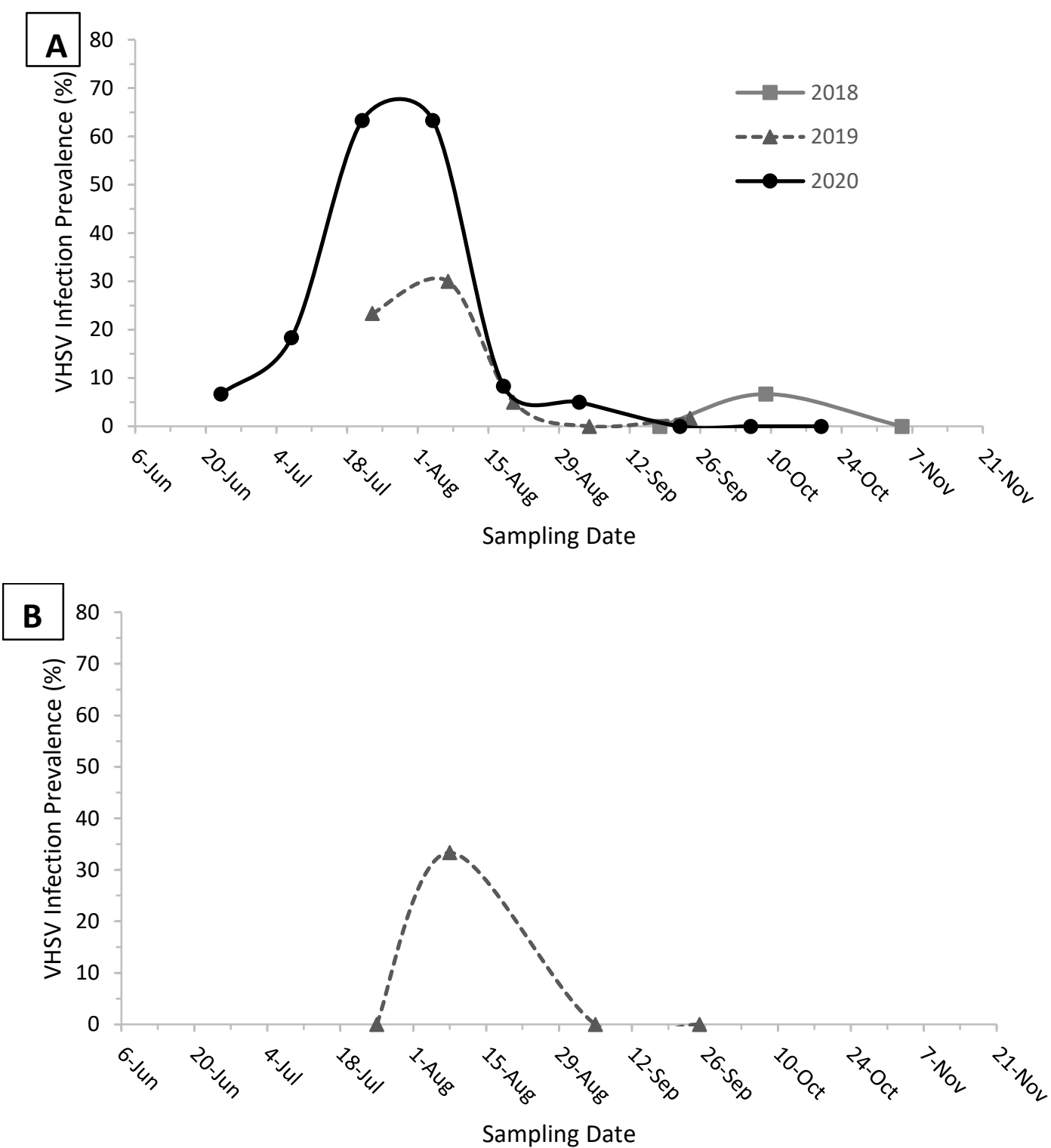

Figure 1. Progression of VHS virus epizootics in age 0 Pacific herring C. pallasii from the Puget Sound, WA region, including (A) Port Angeles Harbor (2018-2020) and (B) Port Ludlow Harbor (2019). Each data point reflects $n=30-60$ fish.

\section{Results}

The typical endemic phase of VHS virus occurred during most sampling events in the period 2001-2020, when positive virus samples were detected in only 9/6277 Pacific herring C. pallasii from 8/117 independent sample collections (Table 1). During these endemic periods, viral titers in kidney/spleen pools from positive samples were at or below the minimum threshold of the plaque assay, and virus isolations could generally be confirmed only after blind passage onto fresh cells.

Localized VHS epizootics in juvenile Pacific herring C. pallasii also occurred throughout the coastal areas of the NE Pacific Ocean, with some outbreaks occurring in the same locations in consecutive years (Table 2). An epizootic was detected in Sitka Sound, AK, USA (Bear Cove Bay) during March 2011 and was characterized by high infection prevalence $(63 \%)$ and geometric mean tissue titer $\left(6.8 \times 10^{3} \mathrm{PFU} / \mathrm{g}\right)$ among positive samples. The fish were randomly sampled and dead/moribund individuals were not noted. This epizootic occurred at a time when cross sectional sampling indicated that adult conspecifics failed to 
test positive from Long Island, AK, USA, approximately $12 \mathrm{~km}$ away (Table 1). Another epizootic was detected in the San Juan Islands region of northern Puget Sound, WA during September 2014. Pacific herring C. pallasii with external signs of VHS, including focal and petechial hemorrhaging along the flank and fin bases were detected during beach seining sampling. Visual examinations estimated that lesions (Figure 2) occurred on approximately $10 \%$ of the fish during the peak of the outbreak. Samples reported in Table 2 were collected after the peak, when VHS virus was detected from 13 to $27 \%$ of the lesioned fish that were stored at $-20{ }^{\circ} \mathrm{C}$, a sub-optimal condition for virus isolation. A third epizootic was detected in Port Ludlow Harbor, Puget Sound, WA, USA, on 8 August 2019, when 33\% of juveniles tested positive for VHSV. That outbreak was short lived, as samples from the same location failed to test positive either $14 \mathrm{~d}$ before or $28 \mathrm{~d}$ after this event (Figure 1B). Recurring epizootics occurred among newly-metamorphosed juveniles in Hot Spring Cove, BC, Canada (June 2018 and 2019) and Port Angeles Harbor, WA, USA (summer 2019 and 2020). Longitudinal sampling from the later site indicated that protracted epizootics occurred in 2019 and 2020. Sampling in 2018 commenced in September, after the epizootic would have been expected to subside based on data from the subsequent years (Figure 1A). Sampling in 2019 commenced on 23 July, just prior to the disease peak on 7 August, which was characterized by $30 \%$ infection prevalence and geometric mean viral tissue titers of $9.4 \times 10^{4} \mathrm{PFU} / \mathrm{g}$. Sampling in 2020 began even earlier, when larvae were metamorphosing to juveniles; infection prevalence increased from $6.7 \%$ on 23 June peaked at $63 \%$ from 21 July to 4 August, and declined to $0 \%$ by 22 September (Figure 1A); geometric mean viral tissue titers were $1.2 \times 10^{4} \mathrm{PFU} / \mathrm{g}$ during the peak.

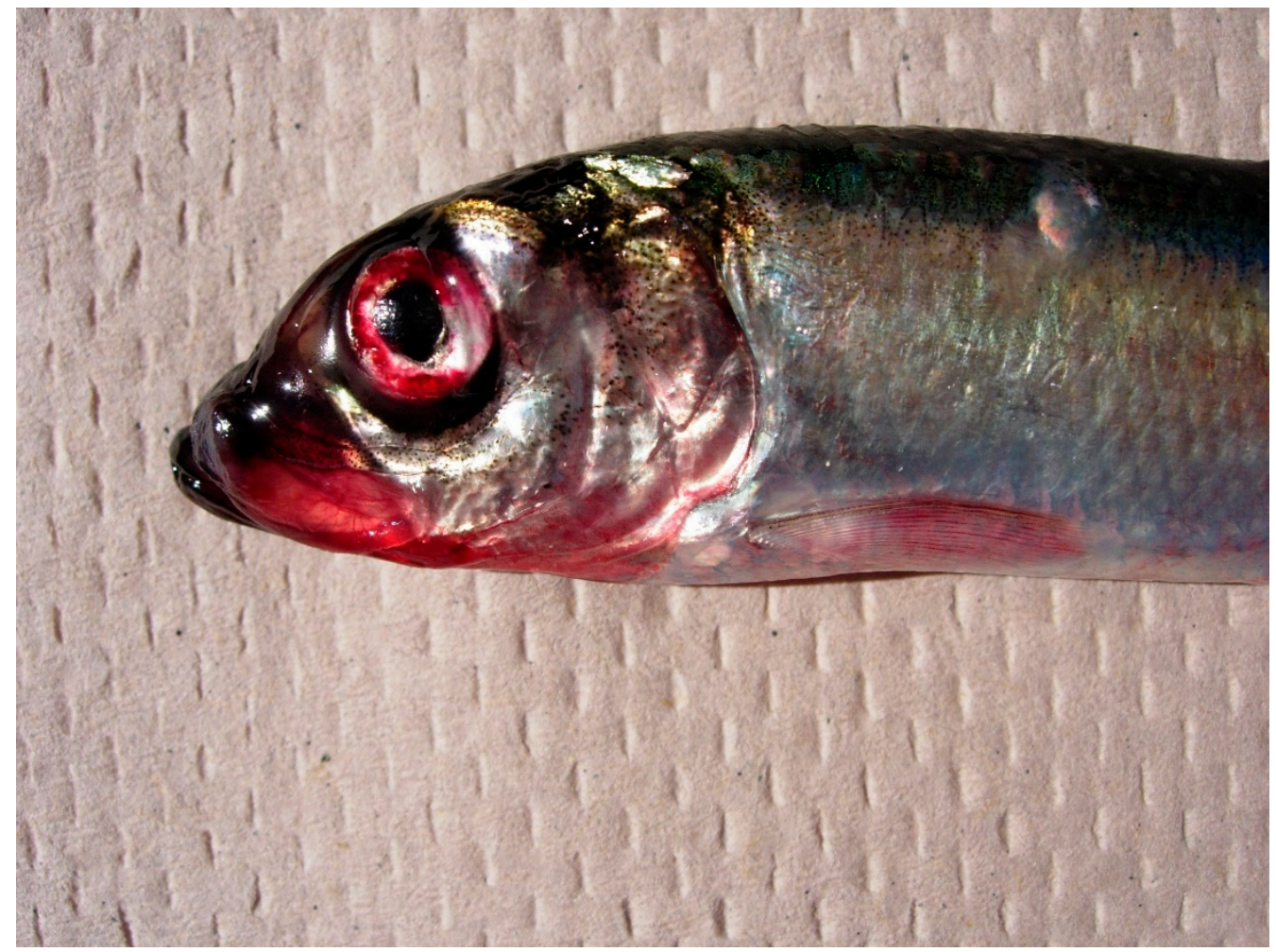

Figure 2. Pacific herring C. pallasii with gross external signs of VHS, including exopthalmia and hemorrhaging around the mouth, eyes, and fins.

\section{Discussion}

Results from 20 years of surveillance efforts indicate that attempted VHS virus isolations from opportunistically collected adult Pacific herring C. pallasii largely return negative results (Table 1), and this traditional biosurveillance approach offers limited value for locating epizootics or informing disease management decisions. Despite these largely negative 
results, these apparently healthy individuals serve as a collective host reservoir by covertly perpetuating VHS virus [5], as outbreaks can often be stimulated in these individuals after their capture, transfer, and confinement [6,7]. Unfortunately, this traditional biosurveillance approach, involving attempted virus isolation, falls short of being able to deduce covert VHS virus carrier states, individual exposure histories, or population impacts of the disease. These results emphasize the need to consider the epidemiology of each disease and define the objectives of field surveillances before committing to survey design and laboratory diagnostics. For example, when attempting to incorporate the impacts of VHS into population assessment models, serological surveillances that deduce exposure histories may provide more informative than traditional virus isolation techniques. Additionally, recent investigations into VHS virus perpetuation mechanisms indicate that future efforts to deduce VHSV exposure histories in Pacific herring C. pallasii may be better served using alternative diagnostic techniques and tissues (i.e., RT qPCR on gill tissues) that provide a much more accurate view of exposure history than attempted virus isolation from kidney/spleen homogenates [5].

Unlike random or opportunistic surveillances in adult Pacific herring C. pallasii, which largely returned negative results, targeted surveillances in juvenile cohorts effectively identified periodic VHS epizootics, some of which recurred at the same time and locations in multiple years (Table 2). Some epizootics occurred over short durations and were detected only at a single timepoint in longitudinal assessments (e.g., Port Ludlow Bay in 2019), and others were more protracted, lasting several months (e.g., Port Angeles Harbor 2020; Figure 1). These differences in disease kinetics may reflect differences in site-specific difference in fish density and behavioral vagility. The host and environmental disease cofactors associated with these epizootics remain unexplored; however, several site-specific observations were common among all outbreaks. First, all the epizootics occurred among juvenile herring, and most (except for Sitka Sound in 2011, which involved age 1+ cohorts) occurred shortly after larval metamorphosis to the juvenile life stage. Second, all epizootics were detected in nearshore areas of embayments or boat harbors containing limited water exchange, where juvenile cohorts occurred in high abundances for extended periods. Finally, although mass mortalities were generally not observed in association with the epizootics (apart from Hot Springs Cove, 2018 and 2019), the outbreaks likely contributed to elevated mortality by predation, as birds and marine mammals were observed consuming distressed fish that were demonstrating abnormal behaviors such as whirling on their longitudinal axes. When combined, these commonalities suggest that some sites are more conducive to VHS outbreaks than others, and that small-scale VHS epizootics in Pacific herring C. pallasii occur more frequently in nearshore areas than previously appreciated. This observation is extended to geographical areas such as Puget Sound, WA, USA, where the virus was previously known to exist, but epizootics were never previously reported in free-ranging wild fish [8], presumably because prior VHS virus surveillances were not directed towards high probability locations and times. These documented singular and recurring epizootics in understudied nearshore habitats suggest that the population-level impacts of VHS to Pacific herring C. pallasii are likely to be underestimated until targeted wild fish surveillances are directed towards the specific times and geographic locations that are most permissive for the disease.

The demonstrated frequency of VHS epizootics among age 0 Pacific herring C. pallasii in nearshore habitats raises questions regarding the accuracy of host/pathogen coevolution paradigms in the context of a highly virulent pathogen like VHS virus. For example, in the "avirulence hypothesis", virulence is assumed to be a consequence of the pathogen's maladaptation to a new host and environment; as such, virulence should be expected to attenuate in long standing host/pathogen relationships [14]. However, VHS virus has likely occurred in the NE Pacific Ocean for several centuries [15], where it has a broad host range that includes a long-standing relationship with Pacific herring C. pallasii. Despite this long-term sympatry, epizootics continue to occur and adaptation towards avirulence or resistant host phenotypes has not occurred. Because there is no published evidence of 
cross-generational transfer of antibodies, annual outbreaks in age 0 cohorts likely occur due to the annual influx of naïve susceptible individuals into the population via larval metamorphosis. This example fails to support the "avirulence hypothesis" and provides support for the more contemporary "trade-off hypothesis" [16,17], whereby the pathogen cost for maintaining fitness through higher virulence can be out-weighed by certain benefits, such as increased transmission with shortened infection duration. This second hypothesis more appropriately explains the Pacific herring C. pallasii/VHS virus interactions, where virulence is maintained concomitantly with high shedding and transmission rates [18] and is analogous to the maintenance of infectious hematopoietic necrosis virus (IHNV) virulence in salmonids [19]. This perpetuation of VHS virus virulence in populations with long-standing host/pathogen co-evolution suggests that epizootics are likely to continue in many regions, including the NE Pacific Ocean, and in other geographic areas such as the Laurentian Great Lakes, where a different VHS virus lineage (Genotype IVb) recently emerged [20].

Shortly after their metamorphosis from larvae to juveniles, age 0 Pacific herring $C$. pallasii adopt a portfolio of life history strategies, whereby some remain in nearshore zones and others quickly venture into more open water pelagic habitats. An immediate reduction in survival potential may be incurred among those utilizing the former habitat, owing to decreased growth rates (unpublished data) and higher potential for VHS outbreaks compared to those subscribing to the open water phenotype. This immediate reduction in survival potential may be offset by long term immunological gains among the survivors, which generate adaptive resistance against future outbreaks of the disease [21]. Future research would be helpful to address this herd immunity hypothesis.

Author Contributions: The concept of recurring disease hot spots was conceived through discussions between P.K.H. and K.A.G. after independent observations supported the same con-clusion. This study was supervised by P.K.H., who also prepared the original draft manuscript. Responsibilities for field collections, laboratory analyses, and manuscript reviewing and editing were shared by P.K.H., T.R.M., J.L.G., M.L.G., S.A.H., H.T.J., A.H.M., A.S.N., E.N.P. and K.A.G. All authors have read and agreed to the published version of the manuscript.

Funding: Funding was provided by the Exxon Valdez Oil Spill Trustee Council, project number 21120111-E, and the U.S. Geological Survey, Ecosystems Program.

Institutional Review Board Statement: This study was conducted according to the guidelines of the Declaration of Helsinki, and approved by the Institutional Animal Use and Care Committee (IACUC) at the Western Fisheries Research Center, Protocol \# 2008-30 (approved 12 December 2013) and 2008-51 (approved 18 May 2017).

Data Availability Statement: The data that support the findings of this study are openly available at https:/ / doi.org/10.5066/P9F55KEO (accessed on 12 July 2021).

Acknowledgments: This study was performed in collaboration with Pacific herring C. pallasii stock assessments in Alaska and Washington. We gratefully acknowledge the field support of Alaska Department of Fish and Game (Stormy Haught, Steve Moffitt, Eric Coonradt, Aaron Dupuis, and Ted Otis), Washington Department of Fish and Wildlife (Todd Sandell, Adam Lindquist, and Kurt Stick), and KWIÁHT (Russel Barsh) and the laboratory support of U.S. Geological Survey (Western Fisheries Research Center and Marrowstone Marine Field Station).

Conflicts of Interest: The authors declare no conflict of interest. The funders had no role in the design of the study; in the collection, analyses, or interpretation of data; in the writing of the manuscript, or in the decision to publish the results. Any use of trade, firm, or product names is for descriptive purposes only and does not imply endorsement by the U.S. Government. 


\section{References}

1. Isshiki, I.; Nishizawa, T.; Kobayashi, T.; Nagano, T.; Miyazaki, Y. An outbreak of VHSV (viral haemorrhagic septicemia virus) infection in farmed Japanese flounder Paralichthys olivaceus in Japan. Dis. Aquat. Org. 2001, 47, 87-99. [CrossRef] [PubMed]

2. Kim, W.S.; Kim, S.R.; Kim, D.; Kim, J.O.; Park, M.A.; Kitamura, S.I.; Kim, H.Y.; Kim, D.H.; Han, H.J.; Jung, S.J.; et al. An outbreak of VHSV (viral hemorrhagic septicemia virus) infection in farmed olive flounder Paralichthys olivaceus in Korea. Aquaculture 2009, 296, 165-168. [CrossRef]

3. Garver, K.A.; Traxler, G.S.; Hawley, L.M.; Richard, J.; Ross, J.; Lovy, J. Molecular epidemiology of viral haemorrhagic septicaemia virus (VHSV) in British Columbia, Canada, reveals transmission from wild to farmed fish. Dis. Aquat. Org. 2013, 104, 93-104. [CrossRef] [PubMed]

4. Meyers, T.R.; Short, S.; Lipson, K. Isolation of the North American strain of viral hemorrhagic septicemia virus (VHSV) associated with epizootic mortality in two new host species of Alaskan marine fishes. Dis. Aquat. Org. 1999, 38, 81-86. [CrossRef] [PubMed]

5. Hershberger, P.K.; MacKenzie, A.H.; Gregg, J.L.; Wilmot, M.D.; Powers, R.L.; Purcell, M.K. Long-term shedding and asymptomatic carriers indicate that Pacific herring are a marine reservoir for viral hemorrhagic septicemia virus. Dis. Aquat. Org. 2021, 144, 245-252. [CrossRef] [PubMed]

6. Hershberger, P.K.; Kocan, R.M.; Elder, N.E.; Meyers, T.R.; Winton, J.R. Epizootiology of viral hemorrhagic septicemia virus in herring from the closed pound spawn-on-kelp fishery. Dis. Aquat. Org. 1999, 37, 23-31. [CrossRef] [PubMed]

7. Kocan, R.M.; Hershberger, P.K.; Elder, N.E.; Winton, J.R. Epidemiology of viral hemorrhagic septicemia (VHS) among juvenile Pacific herring and Pacific sand lances in Puget Sound, Washington. J. Aquat. Anim. Health 2001, 13, 77-85. [CrossRef]

8. Hershberger, P.K.; Gregg, J.; Pacheco, C.; Winton, J.; Richard, J.; Traxler, G. Larval Pacific herring, Clupea pallasii (Valenciennes), are highly susceptible to viral hemorrhagic septicemia and survivors are partially protected after their metamorphosis to juveniles. $J$. Fish Dis. 2007, 30, 445-458. [CrossRef]

9. $\quad$ Batts, W.N.; Lovy, J.; Getchell, R.; Faisal, M.; Standish, I.; Warg, J.V.; Phelps, N.B.D.; Glenney, G.; Winton, J.R. 2.2.7 Viral Hemorrhagic Septicemia. In Fish Health Section Blue Book-Suggested Procedures for the Detection and Identification of Certain Finfish and Shellfish Pathogens; American Fisheries Society: Bethesda, MD, USA, 2020. Available online: https://units.fisheries.org/fhs/ fish-health-section-blue-book-2020/section-1-diagnostic/ (accessed on 21 January 2021).

10. Batts, W.N.; Winton, J.R. Enhanced detection of infectious hematopoietic necrosis viris and other fish viruses by pretreatment of cell monolayers with polyethylene glycol. J. Aquat. Amim. Health 1989, 1, 284-290. [CrossRef]

11. Fijan, N.; Sulimanović, D.; Bearzotti, M.; Muzinić, D.; Zwillenberg, L.; Chilmonczyk, S.; Vautherot, J.; de Kinkelin, P. Some properties of the epithelioma papulosum cyprini (EPC) cell line from carp Cyprinus carpio. Ann. Inst. Pasteur Virol. 1983, 134, 207-220. [CrossRef]

12. Winton, J.; Batts, W.; de Kinkelin, P.; LeBerre, M.; Bremont, M.; Fijan, N. Current lineages of the epithelioma papulosum cyprini (EPC) cell line are contaminated with Fathead Minnow, Pimephales promelas, cells. J. Fish Dis. 2010, 33, 701-704. [CrossRef]

13. Garver, K.A.; Hawley, L.M.; McLure, C.A.; Schroeder, Y.T.; Aldous, S.; Doig, F.; Snow, M.; Edes, S.; Baynes, C.; Richard, J. Development and validation of a reverse transcription quantitative PCR for universal detection of viral hemorrhagic septicemia virus. Dis. Aquat. Org. 2011, 95, 97-112. [CrossRef] [PubMed]

14. Methot, P.O. Why do parasites harm their host? On the origin and legacy of Theobald Smith's law of "declining virulence"1900-1980. Hist. Philos. Life Sci. 2012, 34, 561-601. [PubMed]

15. Einer-Jensen, K.; Pherns, P.; Forsberg, R.; Lorenzen, N. Evolution of the fish rhabdovirus viral haemorrhagic septicaemia virus. J. Gen. Virol. 2004, 85, 1167-1179. [CrossRef] [PubMed]

16. Anderson, R.M.; May, R.M. Coevolution of hosts and parasites. Parasitology 1982, 85, 411-426. [CrossRef] [PubMed]

17. Alizon, S.; Hurford, A.; Midea, N.; Van Baalen, M. Virulence evolution and the trade-off hypothesis: History, current state of affairs, and the future. J. Evol. Biol. 2009, 22, 245-259. [CrossRef] [PubMed]

18. Hershberger, P.K.; Gregg, J.L.; Grady, C.A.; Hart, L.; Roon, S.E.; Winton, J.R. Factors controlling the early stages of viral hemorrhagic septicemia epizootics: Low exposure levels, virus amplification, and fish-to-fish transmission. J. Fish Dis. 2011, 34, 893-899. [CrossRef]

19. Wargo, A.R.; Garver, K.A.; Kurath, G. Virulence correlates with fitness in vivo for two M group genotypes of infectious hematopoietic necrosis virus (IHNV). Virology 2010, 404, 51-58. [CrossRef] [PubMed]

20. Kim, R.; Faisal, M. Emergence and resurgence of the viral hemorrhagic septicemia virus (Novirhabdovirus, Rhabdoviridae, Mononegavirales). J. Adv. Res. 2011, 2, 9-23. [CrossRef]

21. Hershberger, P.K.; Garver, K.A.; Winton, J.R. Principles underlying the epizootiology of viral hemorrhagic septicemia in Pacific herring and other fishes throughout the North Pacific Ocean. Can. J. Fish. Aquat. Sci. 2016, 73, 853-859. [CrossRef] 